\title{
Effect of a Worksite Walking Competition on Health- Related Quality of Life Among University Employees
}

\author{
Breanna Z. Orozco*, Lisa J. Leininger, and Kendra L. Contente \\ Department of Kinesiology, California State University, Monterey Bay, CA \\ Students: borozco@csumb.edu*,kcontente@csumb.edu \\ Mentor:lleininger@csumb.edu
}

\begin{abstract}
Worksite health promotion programs (WHPPs) aim to improve the health and wellness of employees in an effort to improve health related quality of life (HRQOL). The effect of exercise on improving HRQOL is well documented among clinical populations. However, few studies have examined the effect of WHPPS on HRQOL. The purpose of this study was to investigate the effect of a six-week "Workplace Walk-Off Competition" (WWC) on HRQOL among university employees. One hundred and nine university employees were included in this study (WWC group: $n=47$, Control group: $n=62$ ). All study participants completed the Short Form 12 Question, Version 2 (SF-12v2), a HRQOL questionnaire, before and after the WWC. The SF-12v2 questionnaire determines HRQOL based on two components and reports scores for a physical component summary (PCS) score and a mental component summary (MCS) score. A two-way repeated measures ANOVA was performed on PCS and MCS scores, followed by dependent t-tests for each group. There was no significant difference in PCS or MCS scores between the groups. Further, there were no statistically significant changes in PCS or MCS scores ( $p>$.05) among either group, following the six-week WWC. Although much research deems WHPPS effective for improving many health indicators, this short-term program was not effective in improving PCS and MCS components of HRQOL.
\end{abstract}

\section{KEYWORDS}

Quality of Life, Health Promotion, Walking Competition, University Employees

\section{INTRODUCTION}

According to the American Time Survey for 2012, Americans spend a significant amount of their day at work, averaging a total of 8.8 hours. ${ }^{1}$ The significant time spent at the workplace creates an ideal setting for structuring health behavior initiatives, such as worksite health promotion programs (WHPPs). Research demonstrates WHPPs focused on physical activity offer benefits for both the employee and the organization. Individual employee benefits include improvements in health indicators such as body mass index, perceived stress, cholesterol, blood pressure levels and physical activity amounts. ${ }^{2-6}$ Organizational benefits include decreases in absenteeism, increases in job productivity, and substantial health care savings. ${ }^{7,8}$

One of the primary goals of improving individual health indicators, including physical activity, is to improve perceived quality of life. Health-related quality of life (HRQOL) is a multifaceted concept encompassing many health related characteristics. HRQOL dimensions are frequently separated into physical and mental components. ${ }^{9,10}$ Research indicates that HRQOL can be improved with physical activity participation; higher physical activity levels are associated with an increased HRQOL."

Utilization of physical activity programs as a method to improve HRQOL in patients with various chronic diseases are well documented..$^{10,12}$ However, fewer studies have focused on university physical activity programs' effect on HRQOL among employees. A recent study found that 
university employees participating in a four-month walking program increased the mental component ratings of HRQOL, but showed no significant changes in physical component ratings. ${ }^{9}$ Due to the limited and mixed results of the current literature, the purpose of this study was to determine the effect of a six-week worksite walking competition on HRQOL among university employees.

\section{METHODS}

Program Description

The Workplace Walk-Off Competition (WWC) was a six-week walking program open to all employees $(n=878)$ at a small California university. The WWC was a collaborative effort between the Kinesiology and Human Resource Departments. The program was staffed by two Human Resource administrators, one Kinesiology professor, and 10 undergraduate Kinesiology students.

A total of 170 university employees participated in the WWC (95\% of WWC participants completed all components of the program). To provide accountability and foster comradery, competition participants self-assembled into teams of five (for a total of 34 teams). Pedometers were used to quantify physical activity amounts. Each team member recorded their total number of steps per week onto a website designed and maintained by program staff. The WWC included a "Kick Off" event, three "Lunch \& Learn" meetings, and a final awards ceremony. During the "Kick Off" event researchers explained the rules of the WWC and introduced the teams. The "Lunch \& Learns" served as an educational component of the competition and were presented by the Kinesiology professor and included topics such as, "Choose This, Not That," "Healthy Shopping and Cooking for the Family," and "Healthy Living for the Busy Family." In an effort to encourage an increase of step counts, WWC facilitators set weekly step goals for WWC participants. The weekly step goal began at 30,000 steps for week one and gradually increased by 10,000 steps each subsequent week. All participants in the WWC, who met the step goal for the week, were entered into a weekly prize drawing. Weekly prizes were donated by local businesses.

\section{Participants}

One week prior to the start of the WWC, all university employees were sent an email soliciting participation in an online survey. Study procedures and information were provided to all participants of the study, and informed consent was obtained. Approval to conduct the study was granted by the University's Human Subjects Institutional Review Board. One week following the WWC, the same survey was sent via email. A total of 109 employees completed both surveys (WWC group $=62$, control group $=47$ ).

\section{Protocol}

This study was a pre/post design, including a WWC group and control group. The WWC group included individuals who participated in the six-week WWC. The control group consisted of employees who chose not to participate in the WWC.

Data were collected the week prior to the start, and the week following, the WWC. The Short Form, 12 Question, Version 2 (SF 12v2) questionnaire was used to assess HRQOL. The SF-12v2 questionnaire was administered via an online link.

The SF-12v2 questionnaire is a validated and reliable health survey including 12 -questions. ${ }^{13}$ For example, one question of the SF-12v2 reads "During the past 4 weeks, how much of the time has your physical health or emotional problems interfered with your social activities (like visiting with friends, relatives, etc.)?" The SF-12v2 questionnaire is divided into two quality of life components, the Mental Component Summary (MCS) and Physical Component Summary (PCS). Each

\section{AJUR Volume 12 | Issue 3 | May 2015}


component is then divided into an additional four categories. The MCS is divided into vitality, social functioning, role-emotional and mental health. The PCS is divided into physical functioning, role physical, bodily pain, and general health. The MCS and PCS are scored from $0-100$, using norm-based methods. A linear t-score transformation method was used so that both the PCS and MCS have a mean of 50 and a standard deviation of 10 in the general U.S. population (from 1998). ${ }^{13}$ Therefore, a higher score would indicate a higher HRQOL. Health Outcomes Scoring Software (Quality Metric, Lincoln, RI) was used to score the SF-12 questionnaires. To further explore perceived overall health, the authors chose to evaluate one of the questions from the SF 12v2. This question asked the respondent to indicate how they perceived their overall health, with the choices of: Excellent, Very Good, Good, Fair, or Poor.

\section{Statistical Analysis}

A two-way analysis of variance (ANOVA) with repeated measures was used to analyze the PCS and MCS scores. The independent variables for the study were time (pre and post WWC) and participation status (participation in WWC, or no participation in the WWC). Dependent variables were PCS and MCS scores. To assess within-group changes in PCS and MCS scores, t-tests for dependent samples were performed. To further explore perceived overall health, a Chi-Square for linearity was performed for perceived health ratings between groups. Data analyses were conducted using SPSS 21.0. The level of significance was set at $\alpha=.05$.

\section{RESULTS}

Demographics variables were self-reported online following completion of the SF 12v2 questionnaire $(\mathrm{n}=109)$. Demographics of the study participants are shown in Table 1.

\begin{tabular}{|c|c|c|c|c|}
\hline & \multicolumn{2}{|c|}{ Control Group $(n=47)$} & \multicolumn{2}{|c|}{ WWC Group $(n=62)$} \\
\hline & $\mathrm{N}$ & $\%$ & $\mathrm{~N}$ & $\%$ \\
\hline \multicolumn{5}{|l|}{ Gender } \\
\hline Male & 12 & 25.5 & 12 & 19.4 \\
\hline Female & 34 & 72.3 & 49 & 79 \\
\hline Decline to State & 1 & 2.1 & 1 & 1.6 \\
\hline \multicolumn{5}{|l|}{ Ethnicity } \\
\hline Hispanic/Latino & 7 & 14.9 & 7 & 11.3 \\
\hline White & 29 & 61.7 & 40 & 64.5 \\
\hline Other & 9 & 19.1 & 12 & 19.4 \\
\hline Decline to State & 2 & 4.3 & 3 & 4.8 \\
\hline \multicolumn{5}{|l|}{ Job Position } \\
\hline Staff & 26 & 55.3 & 41 & 66.1 \\
\hline Faculty & 17 & 36.2 & 12 & 19.4 \\
\hline Administration & 3 & 6.4 & 8 & 12.9 \\
\hline Decline to State & 1 & 2.1 & 1 & 1.6 \\
\hline
\end{tabular}

Table 1. Study participant demographics. 


\section{PCS Scores}

A two-way repeated measure (2x2) ANOVA was computed with time as the within-subject factor and participation as the between subject factor. There was no significant effect found for time $(\mathrm{p}=.968)$ or an interaction between time and participation $(\mathrm{p}=.542)$. This indicates that there is no difference in pre or post PCS scores between either the control or WWC group.

Results of the paired sample t-test indicated no significant difference $(\mathrm{p}=.624)$ in PCS score pre$(M=51.9 \pm 8.6)$ to post-WWC $(M=52.6 \pm 7.47)$ among the WWC group. Among the control group, there was also no significant difference $(\mathrm{p}=.704)$ in PCS score pre- $(\mathrm{M}=52.5 \pm 7.0)$ to post-WWC $(\mathrm{M}=51.9 \pm 7.4)$. Figure 1 shows mean scores for PCS between the WWC group and the control group.

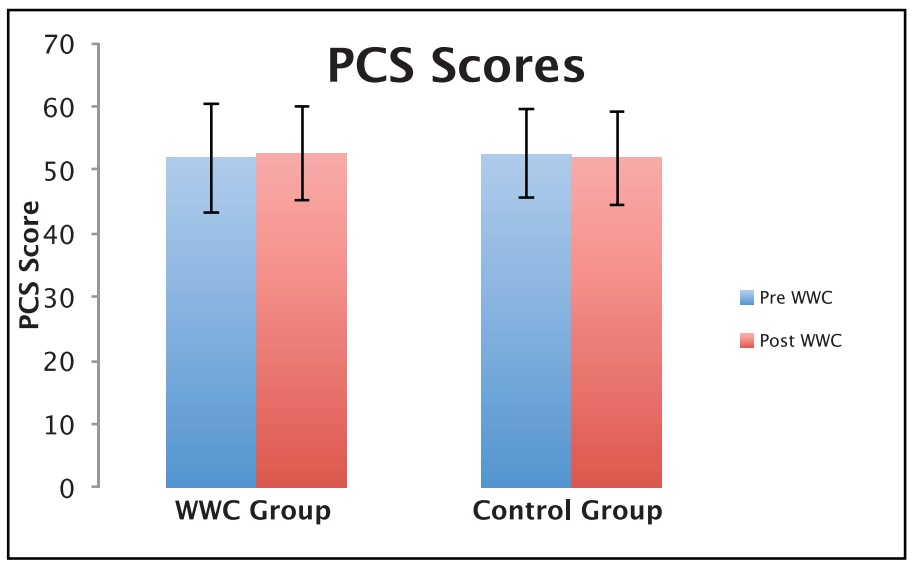

Figure 1. Mean PCS scores pre- and post-WWC between groups.

\section{MCS Scores}

A two-way repeated measure $(2 \times 2)$ ANOVA was computed with time as the within-subject factor, and participation as the between subject factor. There was no significant effect found for time $(\mathrm{p}=.784)$ or an interaction between time and participation $(\mathrm{p}=.631)$.This indicates that there is no difference in pre or post MCS scores between either the control or WWC group.

Results of the paired sample t-test indicate no significant differences $(\mathrm{p}=.885)$ in MCS score pre$(\mathrm{M}=49.4 \pm 8.5)$ to post-WWC $(\mathrm{M}=49.2 \pm 9.6)$ for the WWC group. There were also no significant differences in MCS score pre- $(M=48.8 \pm 8.6)$ to post-WWC $(M=49.7 \pm 8.5)$ for the control group. Figure 2 shows mean scores for MCS for the WWC group and the control group.

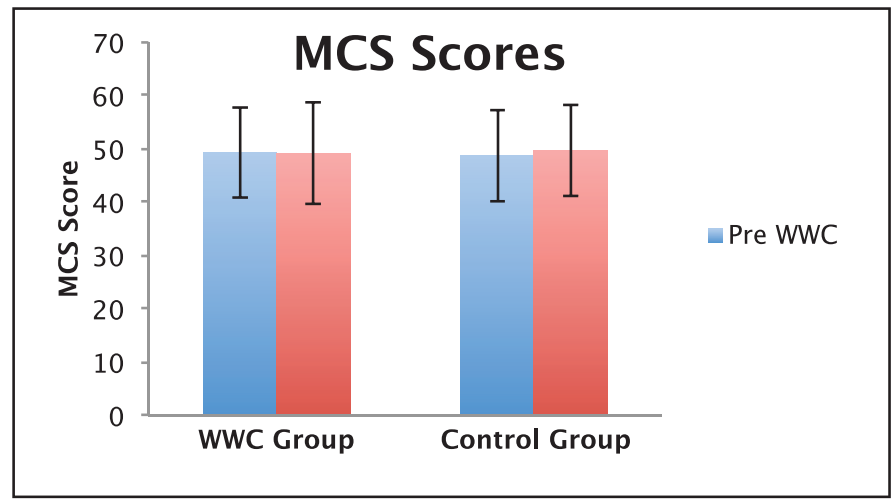

Figure 2. Mean MCS scores pre- and post-WWC between groups. 


\section{Perceived Health Ratings}

As part of the HRQOL survey, study participants were asked to rate their perceived general health on a 1-5 Likert scale (1=Excellent, $2=$ Very Good, $3=$ Good, 4=Fair, or 5=Poor. Figures 3 and 4 report percentages of respondents for each health category, pre- and post-WWC. Most respondents indicated that their health was either "very good" or "good."

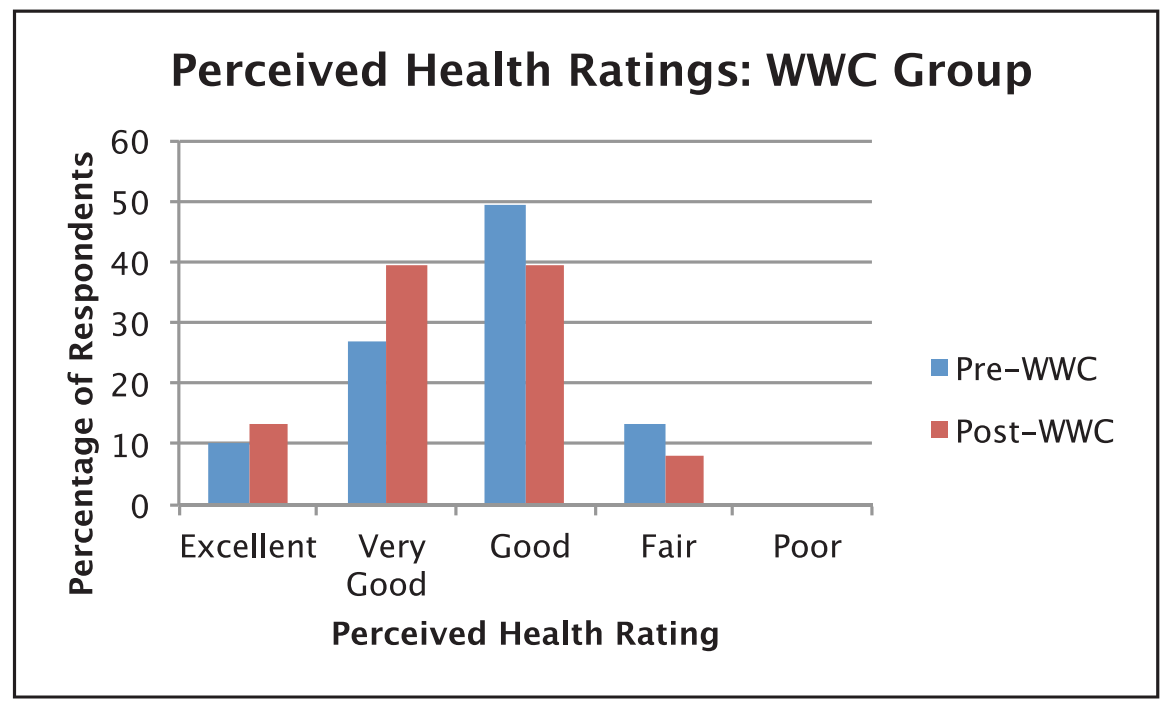

Figure 3. WWC group perceived health ratings pre- and post-WWC.

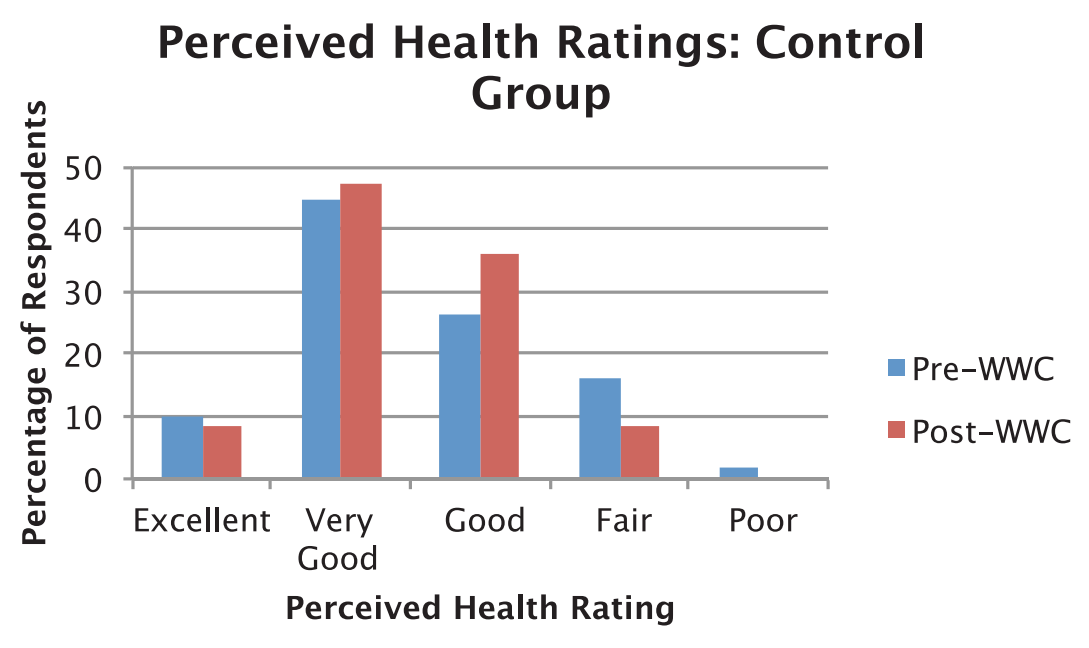

Figure 4. Control group perceived health ratings pre- and post-WWC. 
To further explore the perceived health ratings, A Chi-Square for linearity was performed to assess differences between groups. Results indicate that there were no difference in health rating pre-WWC $\left(\chi^{2}=.41, \mathrm{p}=.53\right)$ between the WWC and control groups. Further, there were no differences in health rating post-WWC $\left(\chi^{2}=.03, \mathrm{p}=.85\right)$ between the WWC and control groups.

\section{DISCUSSION}

The purpose of this study was to examine if HRQOL could improve with participation in a workplace walking competition. The results of this study indicate that the program did not have an effect on HRQOL. Although not statistically significant, there was a small increase in PCS score in the WWC group and a decrease for the control group. However, results were reversed in terms of the MCS scores. MCS scores for the WWC group decreased, while MCS scores for the control group increased. The outcome of this study is consistent with previous findings revealing no statistically significant change in PCS following a worksite health promotion program. ${ }^{9}$

The lack of improvement in HRQOL may be a result of the time of the year this study took place, even though the researchers attempted to address this through use of a control group. The study began during the middle of the academic semester and concluded during the last week of the school year. The end of the semester is a considerably busy time for staff and faculty, as they begin preparation for end of the year tasks. For instance, faculty are immersed in end of the year projects, exams, and finalizing grades. Staff members are just as busy by finishing paper work for graduation, counseling students, or completing other campus projects.

The major limitation of this study was the short time frame of the program. HRQOL is extremely complex and multifaceted. This program focused solely on physical activity participation and did not take an overall holistic approach toward improving health (e.g. nutrition, stress management, etc.) This may indicate that physical activity alone at the worksite is not sufficient to improve HRQOL. Also, the distribution of the WWC group and the control group was not randomized. This was not possible due to the voluntary nature of the WWC and survey solicitation. It may be that those who choose to participate in the study already rate their HRQOL higher in comparison to the rest of the population. Finally, as previously noted, of the $170 \mathrm{WWC}$ participants, 109 (64\%) participated in the study. Thus, the results of this study may not accurately reflect the entire participant population. Further, only 19\% of the employee population participated in the WWC. Therefore, these results may not be generalizable to the entire university population.

Most of the study participants rated their health as either "very good" or "good." It appears that the individuals included in this study have overall perceived good health. Due to this, impact of a short term walking program may be limited. Therefore, future programs and thus, research, should focus on "high-risk" individuals, or those who rate their health as "fair" or "poor." Additionally, future research projects could objectively stratify risk at the onset of programming, due to the fact there may be some difference in what individuals perceive as good health and actual health indicators.

\section{Implications and Areas of Future Research}

This study has many implications for both administrators and participants of WHPPs. Although there are several studies indicating positive health changes for employees (e.g. physical activity level, stress), these results are consistent with previous research indicating little change in HRQOL. Therefore, it may be beneficial for program administrators to weigh the cost versus potential benefits when initiating a program. Further, if a program already exists, an administrator may elect to do a cost-benefit analysis during the evaluation process. Administrators should also be selective in the claims and language they use when marketing the program, and be very precise. For instance, much research indicates that physical activity increases with WHPP participation, so an 
administrator should choose that claim rather than indicating overall HRQOL increases. Additionally, participants should be critical consumers of these programs, and also weigh the cost (whether it be financial, time, etc.) in relation the realistic benefits they may gain. For example, attending a local gym may be a more feasible health practice for their lifestyle, rather than attending programs at the worksite.

Secondly, based on results of this and previous studies, administrators of such programs should increase the duration of programs in order to potentially affect HRQOL. Harding et al. ${ }^{9}$ recently examined a similar worksite walking program. Similar to the WWC used in this study, participants used pedometers, with a 10,000 step per day goal. Harding et al.'s program also had participants report their steps with a program website, and administrators sent monthly encouraging emails, which is nearly identical to this program. Results indicated that the MCS score for HRQOL increased for participants who had participated in the program in repeated years. The major difference between their program and the current study is the duration of the walking program. Harding et al. examined the effects after four months of participation, while this study was conducted over six weeks. Therefore, based on the differences in results, the authors postulate that a longer worksite program may be necessary to illicit increases in HRQOL. If HRQOL is the main goal of the program, administrators may also wish to implement different interventions to improve HRQOL. Due to the complex nature of HRQOL, more comprehensive, longer term programs may be necessary to illicit such increases in HRQOL.

Areas of future research should focus on which components of a WHPP are most effective for improving health indicators. For example, this program had many components: the walking competition, use of teams, use of pedometers, website information, lunch-and-learn presentations, and incentives. All of these components may or may not contribute to the success of similar programs, and thus should be evaluated. Programs of longer duration should also be studied, with perhaps different measurement tools for HRQOL.

In conclusion, our findings indicate that a six-week worksite walking competition had limited impact on HRQOL. However, ample research indicates WHPPs are beneficial for both the individual and organization. Overall, more comprehensive, long-term programs at universities should be studied in an effort to help improve health and quality of life among employees.

\section{REFERENCES}

[1] (2012) American Time Use Survey, In Bureau of Labor Statistics, United States Department of Labor, http://www.bls.gov/tus/datafiles_2012.htm; cited 2014 June 25

[2] Haines, D. J., Davis, L., Rancour, P., Robinson, M., Neel-Wilson, T., and Wagner, S. (2007) A pilot intervention to promote walking and wellness and to improve the health of college faculty and staff, Journal of American College Health: J of ACH 55, 219-225.

[3] Gold, B. C., Burke, S., Pintauro, S., Buzzell, P., and Harvey-Berino, J. (2007) Weight loss on the web: A pilot study comparing a structured behavioral intervention to a commercial program, Obesity $15,155-164$.

[4] Leininger, L., Harris, D., Tracz, S., and Marshall, J. (2013) Differences in physical activity participation between university employees with and without a worksite health promotion program, California Journal of Health Promotion 11, 67-75.

[5] Leininger, L., Orozco, B., and Adams, K. (2014) Worksite based walking competition: effects on perceived stress and physical activity in female university employees, Journal of Fitness Research 3 , $32-38$.

[6] Yang, X., Ge, C., Hu, B., Chi, T., and Wang, L. (2009) Relationship between quality of life and occupational stress among teachers, Public Health 121, 750-766. 
[7] Finkelstein, E. A., Fiebelkorn, I. C., and Wang, G. (2003) National medical spending attributable to overweight and obesity: how much, and who's paying?, Health Affairs Supp/ Web Exclusives, W3219-226.

[8] Tunceli, K., Bradley, C. J., Nerenz, D., Williams, L. K., Pladevall, M., and Elston Lafata, J. (2005) The impact of diabetes on employment and work productivity, Diabetes Care 28, 2662-2667.

[9] Harding, J., Freak-Poli, R. L., Backholer, K., and Peeters, A. (2013) Change in health-related quality of life amongst participants in a 4-month pedometer-based workplace health program, Journal of Physical Activity \& Health 10, 533-543.

[10] Yeo, T., Burrell, S., Sauter, P., Kennedy, E., Lavu, H., Leiby, B., and Yeo, C. (2012) A progressive post resection walking program significantly improves fatigue and health-related quality of life in pancreas and periampullary cancer patients, J Am Coll Surg 214, 463-475.

[11] Anokye, N. K., Trueman, P., Green, C., Pavey, T. G., and Taylor, R. S. (2012) Physical activity and health related quality of life, BMC Public Health 12, 624.

[12] Courneya, K. S., Mackey, J. R., Bell, G. J., Jones, L. W., Field, C. J., and Fairey, A. S. (2003) Randomized controlled trial of exercise training in postmenopausal breast cancer survivors: cardiopulmonary and quality of life outcomes, Journal of clinical oncology: Official Journal of the American Society of Clinical Oncology 21, 1660-1668.

[13] Ware, J., Kosinski, M., Turner-Bowker, D., and Gandek, B. (2002) User's manual for the SF-12v2 health survey with a supplement documenting SF-12 health survey, QualityMetric Incorporated, Lincoln, RI.

\section{ABOUT THE STUDENT AUTHORS}

Breanna Orozco will graduate from California State University, Monterey Bay in Spring 2015 with a Bachelor's degree in Kinesiology-Exercise Science and a Minor in Statistics. Breanna is a McNair scholar, and has received many other prestigious awards, including the American College of Sports Medicine Golding Scholarship. Each summer, Breanna travels to the University of Southern California to work in the Women's Health and Exercise Laboratory. She has presented at many national conferences including the American College of Sports Medicine Annual meetings, and the Hawaiian International Conference on Education. Her future endeavors include pursuing a Ph.D. in Biokinesiology and a career as a research professor.

Kendra Contente will graduate from California State University, Monterey Bay in Spring 2015 with a Bachelor's degree in Kinesiology - Exercise Science. Kendra has been involved in many projects at CSU Monterey Bay, including the annual Workplace Walkoff Challenge. Kendra will be presenting at several national conferences in the next year. Kendra is interested in health and exercise, and her main focus of research for the upcoming year will be interventions to increase physical activity among employees at universities. Kendra plans to pursue medical school.

\section{PRESS SUMMARY}

Research indicates that exercise can help improve health-related quality of life, which adds to the importance of participating in regular physical activity. Worksite health promotion programs aim to improve the health of employees, in an effort to reduce individual disease risk and benefit the organization by reducing health care costs. This study examined if quality of life could be improved among employees with participation in a walking competition at a university. This study did not find that quality of life was improved, however there are many other benefits to worksite health promotion programs, and therefore, studies should continue in this field. 